\title{
The extensional viscosity of a dilute suspension of spherical particles at intermediate microscale Reynolds numbers
}

\author{
By G. RYSKIN \\ Chemical Engineering 208-41, California Institute of Technology, \\ Pasadena, California 91125 \\ With an appendix by \\ G. RYSKIN \\ AND J. M. RALLISON \\ Department of Applied Mathematics and Theoretical Physics, \\ University of Cambridge
}

(Received 29 January 1979 and in revised form 18 January 1980)

The extensional viscosity of a dilute suspension of spherical particles (rigid spheres, viscous drops or gas bubbles) is computed for the case when the Reynolds number of the microscale disturbance motion $R$ is not restricted to be small, as in the classical analysis of Einstein and Taylor. However, the present theory is restricted to steady axisymmetric pure straining flow (uniaxial extension). The rate of energy dissipation is expressed using the Bobyleff-Forsythe formula and then conditionally convergent integrals are removed explicitly. The problem is thereby reduced to a determination of the flow around a particle, subject to pure straining at infinity, followed (for rigid particles) by an evaluation of the volume integral of the vorticity squared. In the case of fluid particles, further integrals over the volume and surface of the particle are required. In the present paper, results are obtained numerically for $1 \leqslant R \leqslant 1000$ for a rigid sphere, for a drop whose viscosity is equal to the viscosity of the ambient fluid, and for an inviscid drop (gas bubble). For the last case, limiting results are also obtained for $R \rightarrow \infty$ using Levich's approach.

All of these results show a strain-thickening behaviour which increases with the viscosity of the particle. The possibility of experimental verification of the results, which is complicated by the inapplicability of the approximation of material frameindifference in this case, is discussed.

\section{Introduction}

It is known that the effective viscosity of a suspension which is subjected to a pure straining motion may increase, thus exhibiting the phenomenon which is commonly known as strain-thickening. One possible explanation of this phenomenon for a suspension of axisymmetric prolate particles at low particle Reynolds number is that the flow causes the particles to be aligned with their axes of symmetry parallel to the principal axis of extension where the force dipole strength is a maximum (Batchelor 1974; Hinch \& Leal 1975, 1976).

Strain-thickening may also occur in the case of a dilute suspension of spherical 
particles if the Reynolds number of the microscale motion is not restricted to be very small - the rate of dissipation due to the presence of the particles is a minimum in the flow with negligible inertia forces according to Helmholtz theorem.

A full description of the effective properties of a suspension including inertial effects for the microscale motion is, of course, a formidable problem. Such a suspension behaves as a non-Newtonian fluid even in the case of rigid spherical particles with negligible interaction (Lin, Peery \& Schowalter 1970; Hinch \& Leal 1975). The contribution to the bulk stress due to the presence of the particles in this case contains a nonlinear momentum-flux term (Batchelor 1970), and the resulting bulk constitutive equation is nonlinear in the bulk strain rate.

Such a suspension may also exhibit 'memory', but a discussion of such effects is beyond the scope of the present paper, which is concerned only with steady, pure straining flow with a uniform velocity gradient.

So far, there has been published only one theoretical paper (Lin et al. 1970) in which the effect of inertia on bulk flow properties is studied. The authors consider steady simple shear flow of a dilute suspension of rigid spheres using an asymptotic expansion for small, but non-zero, Reynolds number, to analyse the flow around a particle. Having obtained the velocity field, they calculate the full bulk stress tensor using a general expression given by Batchelor (1970), which contains integrals of tensor products of such quantities as velocity, position, stress, etc.

The present paper deals with the case of small to moderate Reynolds number for one particular case - namely a dilute suspension of spherical particles (rigid spheres, fluid drops or gas bubbles) which is subjected to steady uniaxial pure straining motion with a uniform velocity gradient.

Batchelor (1970) has provided an expression for the full particle stress tensor. For a steady uniaxial extension, however, a single scalar function of rate of strain (the extensional viscosity $\mu^{*}$ ) completely characterizes the constitutive relation. Since the rate of strain $\Gamma$ has the form

$$
\boldsymbol{\Gamma}=e\left(\begin{array}{rrr}
2 & 0 & 0 \\
0 & -1 & 0 \\
0 & 0 & -1
\end{array}\right)
$$

axial symmetry necessarily implies that the stress tensor is coaxial with $\Gamma$, so that the deviatoric stress has the form

$$
\sigma\left(\begin{array}{rrr}
2 & 0 & 0 \\
0 & -1 & 0 \\
0 & 0 & -1
\end{array}\right)
$$

and then $\sigma=2 \mu^{*} e$.

Now it is convenient here to compute $\mu^{*}$ from the additional rate of energy dissipation in the suspension due to the particles. Batchelor has shown that this approach for determining $\mu^{*}$ necessarily gives the correct result in the present case since the spatial distribution of the average 'microscopic kinetic energy due to the inertia effects' is uniform (Batchelor 1970, equation (4.9)). At the same time it is much easier to compute $\mu^{*}$ using this approach because this computation reduces to evaluation of the integrals of two scalar quantities - vorticity squared and surface velocity squared (see (10) below). This method is particularly advantageous if the problem is solved numerically in the stream-function-vorticity form (as is the case in the present work) since the 
evaluation of velocity components and actual stress components, which are needed for Batchelor's (1970) expression, would require the first and second numerical differentiations of the stream function - rather inaccurate operations. Also, the energy dissipation approach enables us to calculate $\mu^{*}$ very easily for a suspension of inviscid drops as $R \rightarrow \infty(\$ 5)$.

\section{Rate of energy dissipation}

We consider a dilute suspension of neutrally buoyant spherical particles. These particles may be rigid spheres, fluid drops or incompressible gas bubbles (inviscid fluid drops). Drops and bubbles may be considered to be held spherical by sufficiently large surface tension. Let us suppose that the motion of the ambient fluid, without particles, would be a homogeneous and steady axisymmetric straining flow, i.e.

$$
\mathbf{u}=\boldsymbol{\Gamma} \cdot \mathbf{r}, \quad \text { in which } \quad \boldsymbol{\Gamma}=e\left(\begin{array}{rrr}
2 & 0 & 0 \\
0 & -1 & 0 \\
0 & 0 & -1
\end{array}\right) \text { and } e>0
$$

We consider an arbitrary volume, $V$, of the suspension which contains a large number, $N$, of particles and calculate the additional contribution to the viscous dissipation within $V$ which is associated with the presence of the particles. The objective, as indicated above, is to use the particle contribution to viscous dissipation to determine an effective viscosity $\mu^{*}$ for the suspension, as a function of the particle Reynolds number.

To calculate the rate of energy dissipation we use the Bobyleff-Forsythe formula (Serrin 1959). For a volume $\tau$ of homogeneous fluid of viscosity $\mu_{i}$ the rate of dissipation $W_{\tau}$ in $\tau$ is given as

$$
W_{\tau}=\mu_{i} \int_{\tau} \zeta^{2} d \tau+2 \mu_{i} \int_{S_{\tau}} \mathbf{n} \cdot[(\mathbf{u} . \nabla) \mathbf{u}] d S_{\tau}
$$

where $S_{\tau}$ is the boundary of $\tau$ with outward unit normal $\mathbf{n}$ and $\zeta$ is the vorticity corresponding to the velocity $\mathbf{u}$. We use subscript $i=1$ to correspond to the fluid inside the particle and $i=2$ to denote the ambient fluid.

In the absence of any particles the rate of dissipation $W^{0}$ in volume $V$ due to a pure straining motion of the ambient fluid is equal to the second integral in (2) taken over the surface $S$ enclosing the fluid volume $V$ (note, $\zeta=0$ in pure straining flow). On the other hand, it may also be expressed in terms of the rate of dissipation in a unit volume (which is constant) multiplied by $V$,

$$
W^{0}=2 \mu_{2}(\mathbf{\Gamma}: \mathbf{\Gamma}) V=12 \mu_{2} e^{2} V .
$$

Let us denote the rate of dissipation of the pure straining motion in a volume equal to that of the particle $\left(\tau_{0}\right)$ as $\Phi_{0}\left(\Phi_{0}=12 \mu_{2} e^{2} \tau_{0}\right)$. We may then write

$$
W^{0}=\Phi_{0} N / \phi
$$

since $N \tau_{0}=V \phi$, where $\phi$ is the volume concentration of particles. In what follows, we consider the limit $\phi \rightarrow 0$.

The presence of the particles will change the flow so that at a long distance from a given particle (at 'infinity') the flow may be written as $\mathbf{\Gamma} \cdot \mathbf{r}+O(\phi)$. 
In the presence of the particles, the rate of dissipation $W^{*}$ in the volume $V$ of the suspension may be written

$$
W^{*}=Q+W^{0}+w_{s}
$$

Here $Q$ is the sum of integrals in (2) which are taken over the volumes inside and outside the particles (where vorticity now exists due to the disturbance flow) and over the surfaces of the particles. Note that $Q$ is not the total particle contribution to the rate of dissipation. The latter is equal to $Q+w_{s}[O(1)$ as $\phi \rightarrow 0]$, where $w_{s}$ is the change in the value of the integral over the surface $S$ [see (2)] due to the presence of the particles.

Now according to the standard approach to dilute suspension theory (Batchelor 1970; Jeffrey 1974), the flow near each particle is that which would exist if the particle alone were immersed in an infinite body of ambient fluid with a uniform velocity gradient (1) at large distances from the particle. Thus, $Q$ may be written in the form

where

$$
Q=N q+O(\phi)
$$

$$
q=\mu_{1} \int_{\tau_{0}} \zeta_{1}^{2} d \tau+2 \mu_{1} \int_{A_{0}} \mathbf{n} \cdot[(\mathbf{u} . \nabla) \mathbf{u}] d A+\mu_{2} \int_{T_{0}} \zeta_{2}^{2} d T-2 \mu_{2} \int_{A_{0}} \mathbf{n} \cdot[(\mathbf{u} . \nabla) \mathbf{u}] d A .
$$

Here, $\tau_{0}$ and $A_{0}$ are the volume and surface of the particle, $T_{0}$ is the volume external to the particle which extends to infinity, $\mathbf{n}$ the outward unit normal to $A_{0}$ (and, hence, the inward normal as far as $T_{0}$ is concerned), and $\mathbf{u}, \zeta_{1}, \zeta_{2}$ the velocity on the surface and the vorticity inside and outside the particle with the motion at infinity obeying (1). The $O(\phi)$ term in (5) corresponds to a twofold source of error: first, the substitution of the actual average motion of infinity $\boldsymbol{\Gamma} \cdot \mathbf{r}+O(\phi)$ by $\boldsymbol{\Gamma} . \mathbf{r}$ and, secondly, the extension of the integration of vorticity squared over the volume $V$ to infinity (i.e. over $T_{0}$ ) which is possible owing to the rapid decrease of the disturbance vorticity with distance from the particle (see Jeffrey 1977). Note that $q$ is independent of $\phi$ in this dilute limit.

Thus we have divided the additional rate of dissipation into two parts and one of these $(Q)$ does not depend at $O(\phi)$ on the shape of the remote boundary $S$. It may, in fact, be calculated from the problem of flow around a single particle. This division is a direct consequence of the Bobyleff-Forsythe formula (2); the first (volume) integral of this formula contains only disturbance quantities which decrease rapidly with distance from a particle, while the second (surface) integral contains products of disturbance quantities and bulk (pure strain) velocity; the latter increases linearly with distance from the particle.

Insofar as the additional rate of dissipation in $V$ may depend on the shape of the boundary $S$, this dependence is concealed in the quantity $w_{s}$. We will see, however, that the effective viscosity of the suspension may be obtained without calculation of $w_{s}$ and therefore the particular choice of $S$ is unimportant.

Following the basic idea of Einstein, the effective viscosity of suspension $\mu^{*}$ is the viscosity of that hypothetical homogeneous fluid $H$ which, being subjected to the same flow at the boundary $S$ as the suspension, has the same rate of dissipation in $V$.

Let us compute the rate of dissipation in the volume $V$ of the fluid $H$ in the same way, using again the Bobyleff-Forsythe formula (2). Since, by definition, the flow of the fluid $H$ at the boundary $S$ is the same as of the suspension, the integral over the 
surface $S$ in (2) yields the same value as for the suspension [see (4)], but is now multiplied by $\mu^{*}$ instead of $\mu_{2}$, i.e.

$$
\frac{\mu^{*}}{\mu_{2}}\left(W^{0}+w_{s}\right)
$$

On the other hand, there are no particles in the fluid $H$, and therefore if we try to compute the quantity analogous to $Q$ for the fluid $H$, i.e. the volume integral of vorticity squared, we obtain [see (5), (6) and following discussion]

$$
Q_{H}=O(\phi)
$$

Note that we cannot assert that $Q_{H}$ is equal to zero since the flow at the boundary $S$ may be incompatible with the irrotational motion of the fluid $H$ in $V$.

Thus we have the rate of dissipation in the volume $V$ of the fluid $H$,

$$
W_{H}=\frac{\mu^{*}}{\mu_{2}}\left(W^{0}+w_{s}\right)+O(\phi),
$$

and, by definition of the fluid $H$,

$$
W^{*}=W_{H}
$$

Hence

$$
Q+W^{0}+w_{s}=\frac{\mu^{*}}{\mu_{2}}\left(W^{0}+w_{s}\right)+O(\phi)
$$

and recalling that $Q=O(1), W^{0}=O(1 / \phi), w_{s}=O(1)$ as $\phi \rightarrow 0$ :

$$
\begin{aligned}
\frac{\mu^{*}}{\mu_{2}} & =\frac{Q+W^{0}+w_{s}-O(\phi)}{W^{0}+w_{s}}=1+\frac{Q-O(\phi)}{W^{0}+w_{s}}=1+\frac{N q+O(\phi)}{W^{0}+w_{s}} \\
& =1+\frac{N q}{W^{0}}\left(1+\frac{O(\phi)}{N q}\right)\left(1+\frac{w_{s}}{W^{0}}\right)^{-1}=1+\frac{N q}{W^{0}}+O\left(\phi^{2}\right) .
\end{aligned}
$$

So $w_{s}$ cancels explicitly if we are interested only in the solution to the first order in $\phi$.

According to (3)

$$
\frac{\mu^{*}}{\mu_{2}}=1+\frac{q}{\Phi_{0}} \phi ; \quad \text { or } \quad \frac{\mu^{*}-\mu_{2}}{\mu_{2} \phi}=\frac{q}{\Phi_{0}}
$$

Here the numerator $q$ in the case of rigid particles is the integral of the squared vorticity of the disturbance motion multiplied by the viscosity of the ambient fluid; for fluid particles $q$ contains also the integral of the squared vorticity inside the particle and integrals over the particle surface which depend on the velocity.

Thus the problem is reduced to obtaining the flow around a particle which is pure straining at infinity, and then determining $q$ from (6). It should be noted that, though the additional rate of dissipation due to the presence of a given particle is independent of $\phi$ and so may be considered as being $O(1)$ as $\phi \rightarrow 0$, it can actually reach large values depending on the microscale Reynolds number (especially for rigid spheres, see §7). These values (and hence the Reynolds number of the microscale motion) should be, of course, restricted sufficiently that the foregoing approximations [see (7)] remain valid. The restriction may be conveniently expressed as the requirement that the relative contribution of the particles to the suspension viscosity $\left(\mu^{*}-\mu_{2}\right) / \mu_{2}$ must be small compared with unity. This simply means that the present approach, being a dilute-suspension one, is valid only when the effect of the particles is a perturbation (as noted in $\S 3$ of Batchelor 1971). 
Though in principle the present work follows the basic idea of Einstein, I would like to indicate the difference between his original approach and the present one. According to Einstein, the total additional rate of dissipation $\left(Q+w_{s}\right.$ in the present notation) should be calculated. This quantity, however, depends on the shape of the remote boundary (via $w_{s}$ ) and therefore the corresponding integral is not absolutely convergent. In addition, the calculation of the average rate of strain in the suspension gives another nonconvergent integral. This requires the choice of some special shape of the remote boundary (spherical, for example), though the final result does not, in fact, depend on this shape because the contributions from the nonconvergent integrals cancel (see Jeffrey \& Acrivos 1976; Jeffrey 1977).

In the present approach these contributions cancel explicitly for an arbitrary shape of the remote boundary before any actual calculations [see (7)].

Note that the method used by Batchelor $(1967, \$ 4.11)$ which reduces the calculation of the additional rate of dissipation to an integration over the surfaces of the particles is applicable only in the case of $R \rightarrow 0$.

A further interesting illustration of the importance of an explicit consideration of the remote boundary contribution was provided recently by O'Brien (1979) who showed how, using an integration over a large 'macroscopic boundary', one can circumvent the convergence difficulties arising in the case of suspension of interacting particles.

Note also that, if the particles are of arbitrary shape and orientation, and interactions are negligible, then, provided we can calculate for each $k$ th particle the quantity $q_{k}$ from (6), all the speculations of this section remain valid and we obtain

$$
\frac{\mu^{*}-\mu_{2}}{\mu_{2} \phi}=\frac{\sum_{k=1}^{N} q_{k}}{\sum_{k=1}^{N} \Phi_{k}}
$$

where $\sum_{k=1}^{N} \Phi_{k}$ is the rate of dissipation of the pure straining motion (1) in a volume equal to the total volume of the particles in $V$.

\section{On the calculation of the surface integrals in (6)}

These integrals may be reduced to simpler form as follows.

We note that $\mathbf{u}$ is tangential to the particle surface. If we denote the unit tangent vector in the direction of the streamline on the particle surface as $\mathbf{t}$, we may write

$$
\mathbf{u}=u_{s} \mathbf{t}, \quad(\mathbf{u} . \nabla) \mathbf{u}=u_{s} \frac{\partial}{\partial s}\left(u_{s} \mathbf{t}\right)=u_{s}^{2} \frac{\partial \mathbf{t}}{\partial s}+u_{s} \mathbf{t} \frac{\partial u_{s}}{\partial s}
$$

where $\partial / \partial s$ is the derivative along the streamline. Then

$$
\mathbf{n} \cdot[(\mathbf{u} . \nabla) \mathbf{u}]=u_{s}^{2} \mathbf{n} \cdot \frac{\partial \mathbf{t}}{\partial s} \text {. }
$$

Now, we know from differential geometry (McConnell 1957) that $\mathbf{n} .(\partial \mathbf{t} / \partial s)=\kappa_{(n)}$, where $\kappa_{(n)}$ is the normal curvature of the surface in the direction of the streamline, which is equal in magnitude to the curvature of the normal plane section of the surface in that direction and is negative if the surface is convex. 
Thus, on the particle surface

So we may write

$$
\mathbf{n} \cdot[(\mathbf{u} . \nabla) \mathbf{u}]=u_{s}^{2} \kappa_{(n)}
$$

$$
q=\mu_{1} \int_{\tau_{0}} \zeta_{1}^{2} d \tau+2\left(\mu_{1}-\mu_{2}\right) \int_{A_{0}} u_{s}^{2} \kappa_{(n)} d A+\mu_{2} \int_{T_{0}} \zeta_{2}^{2} d T
$$

In this paper we deal only with spherical particles. In this case $\kappa_{(n)}$ is constant and equal to $-1 / a$, where $a$ is the particle radius.

\section{The flow around a sphere embedded in a pure straining motion. State- ment of the problem}

Consider a neutrally buoyant spherical particle (rigid sphere, viscous drop or gas bubble) immersed in a Newtonian incompressible fluid motion which satisfies (1) at infinity. We assume in the case of drop or bubble that the influence of surfactants may be neglected, and that surface tension is sufficiently large that the particle remains spherical in the presence of flow.

In order to use dimensionless quantities, we introduce characteristic scales: for length we adopt the particle radius $a$, for velocity $e a$ and for the rate of dissipation in a volume element $\mu_{2} e^{2} a^{3}$.

Then we have in dimensionless form

$$
\begin{aligned}
\Phi_{0} & =16 \pi ; \\
\frac{\mu^{*}-\mu_{2}}{\mu_{2} \phi} & =\frac{q}{16 \pi} \\
q & =\lambda \int_{\tau_{0}} \zeta_{1}^{2} d \tau+2(1-\lambda) \int_{A_{0}} u_{s}^{2} d A+\int_{T_{0}} \zeta_{2}^{2} d T .
\end{aligned}
$$

Here, $\lambda=\mu_{1} / \mu_{2}$ is the viscosity ratio.

In order to evaluate (9), we need first to determine the flow field in the vicinity of the particle. For a spherical polar coordinate system $(r, \theta, \xi)$, with the origin in the centre of the particle and axis $\theta=0$ directed along the axis of symmetry, the governing Navier-Stokes equations may be written in the form

$$
\begin{gathered}
{\left[\frac{\partial \psi_{i}}{\partial \theta} \frac{\partial}{\partial r}\left(\frac{\zeta_{i}}{r \sin \theta}\right)-\frac{\partial \psi_{i}}{\partial r} \frac{\partial}{\partial \theta}\left(\frac{\zeta_{i}}{r \sin \theta}\right)\right] \sin \theta=\frac{4}{R_{i}} E^{2}\left(\zeta_{i} r \sin \theta\right)} \\
E^{2} \psi_{i}+\zeta_{i} r \sin \theta=0 ; \quad E^{2}=\frac{\partial^{2}}{\partial r^{2}}+\frac{\sin \theta}{r^{2}} \frac{\partial}{\partial \theta}\left(\frac{1}{\sin \theta} \frac{\partial}{\partial \theta}\right) ; \quad R_{i}=\frac{e d^{2} \rho}{\mu_{i}} .
\end{gathered}
$$

Here $\psi_{i}$ is the stream function, $d=2 a$, and $\rho$ is the density.

Boundary conditions are

$$
\begin{aligned}
& \left.\psi_{1}\right|_{\substack{r=0, \frac{1}{\theta}, \pi \\
\theta=0, \frac{1}{2} \pi}}=0 ;\left.\quad \psi_{2}\right|_{\substack{r=1, \theta=0, \frac{1}{2} \pi}}=0 ;\left.\quad \psi_{2}\right|_{r \rightarrow \infty} \rightarrow r^{3} \sin ^{2} \theta \cos \theta ; \\
& \left.\zeta_{1}\right|_{\substack{r=0, \theta=0, \frac{1}{2} \pi}}=0 ;\left.\quad \zeta_{2}\right|_{\theta=0, \frac{1}{2} \pi}=0 ;\left.\quad \zeta_{2}\right|_{r \rightarrow \infty} \rightarrow 0 .
\end{aligned}
$$

On the surface of the particle

$$
\left.u_{1}\right|_{r=1}=\left.u_{2}\right|_{r=1}\left(\equiv u_{s}\right) ; \quad \lambda\left(\zeta_{1}-2 u_{s}\right)_{r=1}=\left(\zeta_{2}-2 u_{s}\right)_{r=1}
$$


According to the assumption of neutral buoyancy $R_{1}=R_{2} / \lambda$ (in the limiting cases $\lambda=\infty$, i.e. rigid sphere, and $\lambda=0$, i.e. gas bubble or inviscid drop, $R_{1}$ does not appear in the problem). Thus the problem is determined by two parameters: the viscosity ratio $\lambda$ and a single Reynolds number (based on the density and viscosity of an ambient fluid) which we will therefore denote as $R$ rather than $R_{2}$.

If the problem (11)-(13) is solved for zero Reynolds number, and the result substituted into (10) and (9), we obtain the well-known Einstein-Taylor formula

$$
\left(\mu^{*}-\mu_{2}\right) / \mu_{2} \phi=(2 \cdot 5 \lambda+1) /(\lambda+1) .
$$

\section{Suspension of incompressible gas bubbles (or inviscid drops at large particle Reynolds numbers}

Consider the case $\lambda=0$ and $R \gg 1$. Using in an extensional flow an approach developed by Levich for the translational motion of a gas bubble at high $R$ (see Batchelor $1967, \S 5.14$ ), we may approximately determine $q$ for this case from the irrotational flow around a sphere, which obeys (1) at infinity,

We have in this case

$$
\psi_{2}=\left(r^{3}-\frac{1}{r^{2}}\right) \sin ^{2} \theta \cos \theta
$$

$$
\begin{aligned}
q & =2 \int_{A_{0}} u_{s}^{2} d A=8 \pi \int_{0}^{\frac{1}{2} \pi} u_{s}^{2} \sin \theta d \theta \\
u_{s} & =-5 \sin \theta \cos \theta
\end{aligned}
$$

and finally

$$
\left(\mu^{*}-\mu_{2}\right) / \mu_{2} \phi=\frac{5}{3} \text {. }
$$

Since at low microscale Reynolds number $\left(\mu^{*}-\mu_{2}\right) / \mu_{2} \phi=1$, it may be said that strain-thickening occurs in the case of a suspension of inviscid drops or bubbles, but the full relative increase of the effective viscosity due to the increase of microscale Reynolds number is not more than $\frac{2}{3} \phi$; we also see that, for either sufficiently small or large particle Reynolds numbers, the effective viscosity is independent of strain rate.

\section{Numerical method of solution at intermediate particle Reynolds numbers}

We regard the particle Reynolds number, $R$, as intermediate if it is in the range $1 \leqslant R \leqslant 1000$. In this case we have to solve the problem (11)-(13) numerically. The method which we use here is similar to one used earlier for the problem of translational motion of a spherical drop in a quiescent fluid (Rivkind \& Ryskin 1976; Rivkind, Ryskin \& Fishbein 1976). First, we introduce the disturbance stream function $\hat{\psi}_{2}$ as the unknown in the external region by subtracting the stream function corresponding to the undisturbed pure straining motion $\hat{\psi_{2}}=\psi_{2}-r^{3} \sin ^{2} \theta \cos \theta$; then we transform the external region (by $\hat{r}=1 / r$ ) into the interior of a circle. The advantages of transformations of the last type for numerical computations in infinite regions were recently demonstrated by Grosch \& Orszag (1977).

The resulting equations are solved via the finite-difference ADI scheme of the Peaceman-Rachford type (Ames 1969; Roache 1972), which exhibits second-order accuracy. 
Let us denote the conventional three-point approximations of the second space derivatives as $\delta^{2} / \delta r^{2}, \delta^{2} / \delta \theta^{2}$ and the centred approximations of the first space derivatives as $\delta / \delta r, \delta / \delta \theta$. Both these approximations have second-order accuracy on the uniform grid used in the present study.

Weare interested only in the steady-state solution, but in order to use an ADI scheme we write the vorticity equation from (11) in non-steady form and introduce an artificial 'time' derivative in the stream-function equation

$$
\begin{aligned}
\frac{\partial \zeta_{i}}{\partial t} & =\frac{4}{R_{i}} \frac{E^{2}\left(\zeta_{i} r \sin \theta\right)}{r \sin \theta}-\frac{1}{r}\left[\frac{\partial \psi_{i}}{\partial \theta} \frac{\partial}{\partial r}\left(\frac{\zeta_{i}}{r \sin \theta}\right)-\frac{\partial \psi_{i}}{\partial r} \frac{\partial}{\partial \theta}\left(\frac{\zeta_{i}}{r \sin \theta}\right)\right], \\
\frac{\partial \psi_{i}}{\partial t} & =E^{2}\left(\psi_{i}\right)+\zeta_{i} r \sin \theta
\end{aligned}
$$

Following the Peaceman-Rachford approach the finite-difference scheme in the internal region may be written as (we drop the subscript 1 for simplicity; superscript denotes the iteration)

$$
\begin{aligned}
\frac{\xi-\zeta^{m}}{\tau_{\zeta}} & =\frac{1}{2}\left[\frac{4 \lambda}{R} \frac{\delta^{2} \tilde{\zeta}}{\delta r^{2}}+A^{m} \frac{\delta \xi}{\delta r}+\frac{4 \lambda}{r^{2} R} \frac{\delta^{2} \zeta^{m}}{\delta \theta^{2}}+B^{m} \frac{\delta \zeta^{m}}{\delta \theta}+\frac{C^{m}}{2}\left(\xi+\zeta^{m}\right)\right] \\
\frac{\zeta^{m+1}-\xi}{\tau_{\zeta}} & =\frac{1}{2}\left[\frac{4 \lambda}{R} \frac{\delta^{2} \xi}{\delta r^{2}}+A^{m} \frac{\delta \tilde{\zeta}}{\delta r}+\frac{4 \lambda}{r^{2} R} \frac{\delta^{2} \zeta^{m+1}}{\delta \theta^{2}}+B^{m} \frac{\delta \zeta^{m+1}}{\delta \theta}+\frac{C^{m}}{2}\left(\zeta^{m+1}+\tilde{\zeta}\right)\right] \\
A^{m} & =\frac{1}{r}\left(\frac{8 \lambda}{R}-\frac{1}{r \sin \theta} \frac{\delta \psi^{m}}{\delta \theta}\right) \\
B^{m} & =\frac{1}{r^{2} \sin \theta}\left(\frac{4 \lambda}{R} \cos \theta+\frac{\delta \psi^{m}}{\delta r}\right), \\
C^{m} & =\frac{1}{r^{2} \sin \theta}\left(-\frac{1}{\sin \theta} \frac{4 \lambda}{R}+\frac{1}{r} \frac{\delta \psi^{m}}{\delta \theta}-\frac{\cos \theta}{\sin \theta} \frac{\delta \psi^{m}}{\delta r}\right) \\
\frac{\tilde{\psi}-\psi^{m}}{\tau_{\psi}} & =\frac{1}{2}\left[\frac{\delta^{2} \tilde{\psi}}{\delta r^{2}}+\frac{1}{r^{2}}\left(\frac{\delta^{2} \psi^{m}}{\delta \theta^{2}}-\frac{\cos \theta}{\sin \theta} \frac{\delta \psi^{m}}{\delta \theta}\right)+\zeta^{m+1} r \sin \theta\right], \\
\frac{\psi^{m+1}-\psi}{\tau_{\psi}} & =\frac{1}{2}\left[\frac{\delta^{2} \psi}{\delta r^{2}}+\frac{1}{r^{2}}\left(\frac{\delta^{2} \psi^{m+1}}{\delta \theta^{2}}-\frac{\cos \theta}{\sin \theta} \frac{\delta \psi^{m+1}}{\delta \theta}\right)+\zeta^{m+1} r \sin \theta\right] .
\end{aligned}
$$

Here $\tau_{\zeta}, \tau_{\psi}$ are the iteration parameters ('time' steps). The steady solution is of second-order accuracy in space and does not depend on the 'time' steps, which should be chosen in such a way as to give the fastest (optimal) convergence. The scheme in the external region is analogous.

The conditions (13) of continuity of the tangential velocity and stress components at the particle surface are approximated as follows (superscript denotes the iteration; $\beta=O(1)$ is a scheme parameter):

for $\lambda \leqslant 1$

for $\lambda>1$

$$
\begin{aligned}
& \zeta_{1}^{m+1}=\zeta_{1}^{m}+\beta\left(u_{2}^{m}-u_{1}^{m}\right), \\
& \zeta_{2}^{m+1}=\lambda \zeta_{1}^{m+1}+(1-\lambda)\left(u_{1}^{m}+u_{2}^{m}\right)
\end{aligned}
$$

$$
\begin{aligned}
& \zeta_{2}^{m+1}=\zeta_{2}^{m}+\beta\left(u_{2}^{m}-u_{1}^{m}\right), \\
& \zeta_{1}^{m+1}=\frac{\zeta_{2}^{m+1}}{\lambda}+\frac{\lambda-1}{\lambda}\left(u_{1}^{m}+u_{2}^{m}\right) .
\end{aligned}
$$


This method of approximating boundary conditions at the fluid-fluid interface is an extension of one given by Dorodnitsyn \& Meller (1968) and Israeli (1970) for a solid-fluid interface. It has a simple physical interpretation which dictates the proper choice for the sign of $\beta$. At every stage of the computations at which the continuity of tangential velocity is not satisfied, the local vorticity is adjusted proportionally to the magnitude of the velocity jump, which is itself equal to the strength of the corresponding vortex sheet at the drop surface. The subsequent diffusion and convection of vorticity lead to a smoothing of the velocity discontinuity.

The normal derivatives at the boundaries [e.g. the tangential velocity in (14)] are calculated with second-order accuracy using one-sided three-point formulae. All the computations were performed with a $81 \times 41$ grid.

Some numerical experiments with the scheme outlined above were done using 'frozen' boundary values of the vorticity. The scheme turned out to be stable (at $R \leqslant 1000$ ) for essentially arbitrary 'time' steps (experiments were done for $10^{-3} \leqslant \tau_{\zeta}$, $\tau_{\psi r} \leqslant 10^{2}$ ). It was found that $\tau_{\zeta}=\tau_{\psi}=1$ gave an adequate rate of convergence (several tens of iterations with an essentially arbitrary initial guess). No attempt was made to determine the optimum sequence of parameters (see Ames 1969).

For the entire problem, with the boundary values of the vorticity determined by (14), an adequate rate of convergence was obtained with

$$
\beta=3 \quad \text { and } \quad \tau_{\psi}=1, \quad \tau_{\zeta}=\left\{\begin{array}{rll}
1 & \text { if } & m \leqslant 150 \\
0.1 & \text { if } & m>150
\end{array}\right.
$$

Approximately 200-400 iterations were necessary for convergence, starting from the irrotational initial field.

\section{Numerical results and discussion}

The computations were done for gas bubbles or inviscid drops $(\lambda=0)$, drops of the same viscosity as the ambient fluid $(\lambda=1)$ and rigid spheres $(\lambda=\infty)$ and for Reynolds numbers $R=1,20,100,400,1000$. The results for larger $R(400,1000)$ are, of course, only approximate, since the grid is too crude for these $R$.

Some comments on the assumption of sphericity of the drop seem appropriate here. The deformation of the drop is governed by some dimensionless group which should include surface tension $\gamma$, rate-of-strain magnitude $e$, drop diameter $d$ and some or all of the remaining parameters, viz. viscosities $\mu_{1}$ and $\mu_{2}$ and density $\rho$.

Obviously this deformation group cannot be a product of some powers of Reynolds number and surface tension alone because this product is not dimensionless. This means that, however high the Reynolds number and however low the surface tension, this deformation group can always (in principle) be made arbitrarily small by some choice of the other parameters. Thus the assumption of sphericity can be justified in principle for arbitrary surface tension and Reynolds number.

In practice, of course, the situation is different, since all the parameters lie within some reasonable ranges. Let us consider an example of a drop $(d=0.1 \mathrm{~cm})$ of organic liquid (say, $\mathrm{CCl}_{4}$ ) in water (or vice versa) so that $\mu_{1} \approx \mu_{2} \approx 0.01 \mathrm{~g} \mathrm{~cm}^{-1} \mathrm{~s}^{-1}, \rho$ $\approx 1 \mathrm{~g} \mathrm{~cm}^{-3}$ and $\gamma \approx 50 \mathrm{dyn} \mathrm{cm}^{-1}$. The rate of strain corresponding to $R=1000$ is then $e=1000 \mathrm{~s}^{-1}$. Now, according to the results of drop deformation theory (Taylor 


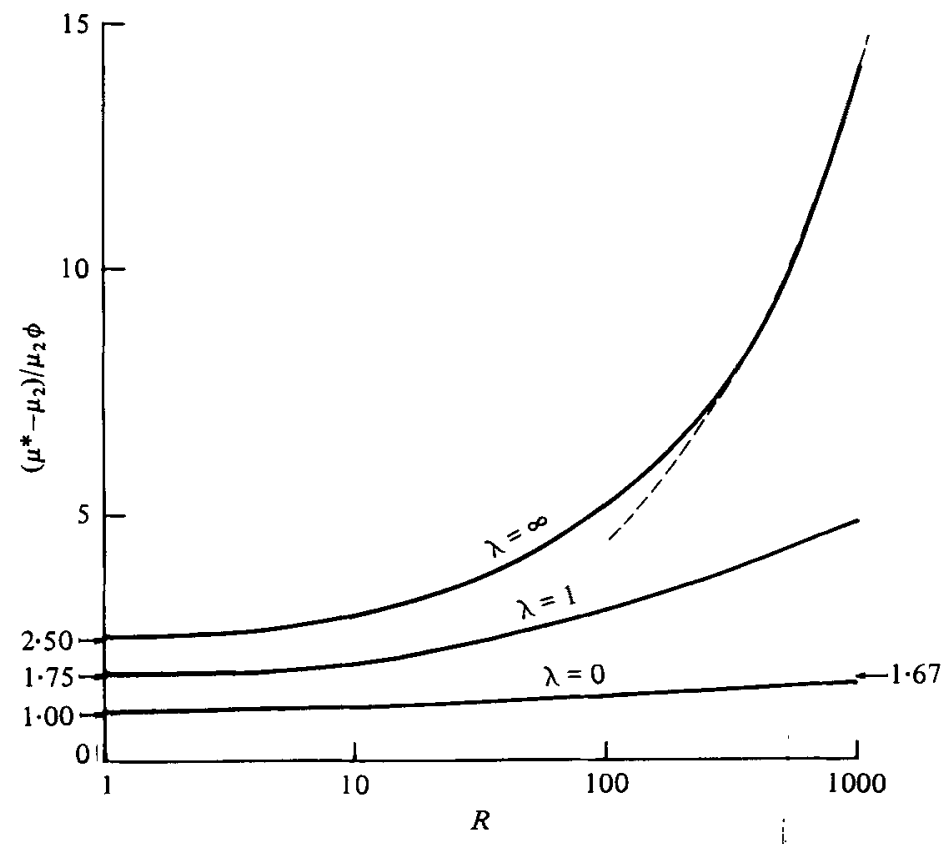

Figure 1. The quantity $\left(\mu^{*}-\mu_{2}\right) / \mu_{2} \phi$ as a function of the microscale Reynolds number. $\lambda=0$, the suspension of inviscid drops; $\lambda=1$, the suspension of drops whose viscosity is equal to the viscosity of the ambient fluid; $\lambda=\infty$, the suspension of rigid spheres. Left-hand arrows: the Einstein and Taylor results for corresponding viscosity ratios $\lambda$; right-hand arrow: the result for the suspension of inviscid drops at $R \gg 1(\S 5)$. Broken line: $0 \cdot 44 R^{\frac{1}{2}}$.

1934), the relative deviation from sphericity is of the same order as the dimensionless group $e d \mu_{2} / \gamma$ and is therefore about $2 \%$ in the considered case, which is acceptable. Note that in the case of high $R$ it would be more likely for deformation to depend upon another dimensionless group, viz. $\rho e^{2} d^{3} / \gamma$, which may change the above conclusion. However, no theory or experiment for this case is currently available.

The results of the computations are shown in figure 1 , plotted in terms of $\left(\mu^{*}-\mu_{2}\right) / \mu_{2} \phi$ versus $R$. The left arrows show the Einstein and Taylor results for the corresponding viscosity ratios $\lambda$. The right arrow shows the result of $\S 5$.

Some predictions of the behaviour of the quantity $\left(\mu^{*}-\mu_{2}\right) / \mu_{2} \phi$ as a function of $R$ (for given $\lambda$ ) can be made on the basis of the Helmholtz minimum dissipation theorem (Batchelor $1967, \S 4.8$ ). In particular, at any $R \neq 0$ this quantity should be larger than at $R=0$ (i.e. larger than the Einstein-Taylor result for the same $\lambda$ ). This means strain-thickening at low Reynolds numbers; however, the monotonicity of this function of $R$ is not guaranteed by the Helmholtz theorem and hence at higher $R$ the strain-thickening is predicted only in a sense of point-wise comparison with the zeroReynolds case.

The numerical results agree with the above predictions and in addition show that a dilute suspension of spherical particles exhibits strain-thickening in the whole intermediate range of strain rates corresponding to values of $R$ in the range $1 \leqslant R \leqslant 1000$. This effect increases with $\lambda$ and becomes rather considerable for rigid spheres.

For this latter case we can estimate $\left(\mu^{*}-\mu_{2}\right) / \mu_{2} \phi$ at large strain rates as follows. According to the present computations of the flow field, there is no noticeable flow 
separation at the particle surface even for rigid sphere at $R=1000$ (the vorticity distribution on the hemisphere surface does not change sign). The reason for this behaviour is, perhaps, the unusual character of the pressure field in the irrotational uniaxial extensional flow over a sphere - though on the sphere surface the pressure, as usual, is increasing from the point of maximum velocity $\left(\theta=45^{\circ}\right)$ to the stagnation point $\left(\theta=0^{\circ}\right)$; this behaviour changes very rapidly with increasing distance from the sphere surface to a situation in which the velocity is increasing from $\theta=45^{\circ}$ to $\theta=0^{\circ}$, and pressure is decreasing. Since the boundary layer for $R \leqslant 1000$ at this part of the sphere surface is not very thin, the imposed pressure at its external boundary is decreasing, and separation does not occur.

Now we can roughly estimate $q$ by means of boundary-layer theory. We estimate the thickness of the boundary layer as $\delta \sim a / R^{\frac{1}{2}}$; the vorticity in it as $\zeta_{2} \sim e a / \delta \sim e R^{\frac{1}{2}}$; the volume of the boundary layer as $a^{2} \delta$ and $q$ as

$$
\begin{gathered}
q \sim \frac{1}{\mu_{2} e^{2} a^{3}} \mu_{2}\left(e R^{\frac{1}{2}}\right)^{2} a^{2} \frac{a}{R^{\frac{1}{2}}} \sim R^{\frac{1}{2}} . \\
\frac{\mu^{*}-\mu_{2}}{\mu_{2} \phi} \sim R^{\frac{1}{2}} .
\end{gathered}
$$

Thus we have

The numerical results for a rigid sphere are approximately proportional to $R^{\frac{1}{2}}$ for larger $R$ (see figure 1), and this fact allows us to estimate the coefficient in (15) as $\approx \mathbf{0 . 4 4}$. This result is indeed very rough but may be useful for correlation of experimental data.

For an inviscid drop (or incompressible gas bubble) the numerical results at $R=1000$ are in good agreement with the asymptotic theory of $\S 5$.

It is interesting that the creeping-flow solutions of Einstein and Taylor turn out to be rather accurate up to $R \approx 1$ - their results differ from the numerical ones by $\approx 1 \%$. For the case of translational motion (Rivkind \& Ryskin 1976; Rivkind et al. 1976) the corresponding difference at $R=1$ is $\approx 10-15 \%$.

It is worth emphasizing that the present results are valid only for a steady axisymmetric pure straining flow. This restricts severely the practical utility of the present quantitative results and complicates the problem of experimental verification. Indeed, insofar as I am aware, there is no data published which could be directly compared with the present results.

The main difficulty is not that the strain rates would need to be large. Indeed, for particles of diameter $10^{-1} \mathrm{~cm}$ in water the necessary rate of strain is in the range from $1 \mathrm{~s}^{-1}$ to $10^{3} \mathrm{~s}^{-1}$, which looks feasible, though the question may arise at this point whether the macroflow will be stable if the microflow has $R=10^{3}$. This question cannot be answered at the moment because there has been no direct investigation of the stability of axisymmetric straining flow. However, Pearson (1959) has stated that 'an axisymmetric contraction is probably the most effective type of contraction for reducing the relative turbulent intensity' and this may suggest that it is more stable than flows with mean vorticity.

The main difficulty is that virtually all achievable viscometric flows are flows with appreciable vorticity (e.g. simple shear flow). In order to understand why this constitutes a problem, let us first discuss some peculiarities of the steady pure straining flow. This flow is steady also in a Lagrangian sense, i.e. in a frame of reference rigidly 
connected with the particle and moving with it along the streamline of the pure straining macroflow (the frame of reference which is, in fact, used in the statement of the problem in \$4), the microflow around the particle is always the same, namely a flow which is a uniaxial extension with fixed magnitude and fixed directions of the principal axes far from the particle. This fact allowed us to look for a single scalar function $\left(\mu^{*}-\mu_{2}\right) / \mu_{2} \phi$ of $\Gamma$ (and thus of $R$ ) and to ignore all other possible effects. Note, for example, that 'memory' effects may appear at non-zero Reynolds number due to the existence of a finite relaxation time for the unsteady microflow comparable to the characteristic time of the macroflow (as $R \rightarrow 0$, the former is the time of vorticity diffusion $\rho d^{2} / \mu_{2}$ and is much smaller than the latter).

The results of Einstein and Taylor for an extensional flow are unchanged by the addition of vorticity since the particles are isotropic, and the microflow is inertialess. At non-zero Reynolds numbers, however, the flows are not superposable (owing to nonlinearity) and thus the present results cannot be verified by shear flow experiments. Note, for example, that simple shear flow is not steady in the Lagrangian sense - the axes of rate-of-strain rotate with respect to the particle. In addition, as shown in the appendix, for a suspension with micro-inertia, the approximation ('principle') of material frame-indifference (Truesdell \& Noll 1965) is also invalid, and therefore experiments in which some part of the fluid is undergoing solid-body rotation in addition to pure strain are also inappropriate, even if the microflow (i.e. pure strain) is steady in the particle frame of reference.

Lin et al. (1970) have presented theoretical results for a suspension with weak inertia in simple shear flow, and their work gives a considerably greater deviation from Einstein's theory for Reynolds numbers of $O(1)$ than is obtained in the present study. It is, of course, possible that this is a simple consequence of applying Lin et al.'s (1970) asymptotic theory at a Reynolds number which is too large. More likely, in my opinion, is the possibility that the difference is a consequence of the presence of vorticity in shear flow. Since superposition of vorticity is not applicable in the presence of microinertia, there is no reason to expect that the two results should be the same at any non-zero Reynolds number.

The author is greatly indebted to Professor L. G. Leal, Professor W. R. Schowalter and Dr J. M. Rallison for the very helpful discussions and comments on the earlier draft of this paper. The author is also most grateful to Professor G. K. Batchelor for a very interesting discussion and to the referees for the useful comments and criticisms.

\section{Appendix. On the applicability of the approximation of material frame- indifference in suspension mechanics}

\section{By G. RYSKIN AND J. M. RALLISON}

The approximation of material frame-indifference (AMFI; usually called 'principle') is frequently invoked in discussion of constitutive relations for non-Newtonian materials (Truesdell \& Noll 1965; Astarita \& Marrucci 1974; Bird, Armstrong \& Hassager 1977; Truesdell 1977; Schowalter 1978). In simple terms, the approximation asserts that the constitutive behaviour of a local material is invariant to rigid-body motions. On the other hand, in at least two problems the AMFI has been shown not to 
be valid: by Lumley (1970), in a discussion of turbulent (but local) constitutive relations; and by Brenner \& Condiff (1974) in considering the transport properties of a suspension of orientable particles in overall rotation (say in a centrifuge). The question therefore arises as to the validity of the AMFI for suspension-like materials in general, and for that discussed in the body of this paper in particular. (If the AMFI is valid, then, as Barthés-Biesel \& Acrivos 1973 have demonstrated, the results from one flow can be generalized to others.)

The AMFI may be stated in two forms, requiring the invariance of the constitutive equation (1) to a superimposed rigid motion of the body (material) or (2) to an arbitrary motion of the observer (Truesdell \& Noll 1965; Truesdell 1977). We will first consider the 'invariance to rigid motion' form.

The instantaneous motion of a macroelement of material (which contains many suspended particles but is nevertheless small in comparison with the suspension as a whole) may be divided into two parts: rigid-body motion (translation and rotation) and pure straining motion. Let us consider the microdynamics (as is commonly done in suspension mechanies) in a frame of reference translating and rotating with the rigid-body motion of the macroelement. The microdynamics in this, in general, noninertial frame of reference is not invariant to a change to an inertial frame of reference (i.e. to a change of rigid-body motion), and so neither is the stress-strain relation for the suspension.

An example is the problem discussed in the body of this paper. The rotation of a macroelement of the suspension will change the structure of the microscale fluid motion around a particle (indeed, if sufficiently rapid it may even generate a Taylor column in the microflow). The disturbance due to the presence of the particles will thus be greater as a result of the rotation and so the quantity $\left(\mu^{*}-\mu_{2}\right) / \mu_{2} \phi$ as measured by the additional energy dissipation will presumably be increased.

Reverting to a discussion of a general suspension, then, it is important to recognize the $a$ priori conditions under which the AMFI is applicable. For the purpose of estimation we consider only one part of the fictitious body force acting in a non-inertial frame (Batchelor 1967, §3.2), namely the Coriolis term. (The terms connected with the translational and centripetal accelerations may be included in a modified pressure for an incompressible fluid.)

Now, for a suspension, there is a fundamental microscale length, $d$ say, associated with the particle size. This is assumed small in comparison with the macroscale $L$. For neglect of the Coriolis term in comparison with viscous forces in determining the stress (i.e. for validity of the AMFI), however, we need $E^{-1} \equiv \Omega d^{2} / \nu \ll 1$, where $\Omega$ is a typical angular velocity of the macroelement with respect to an inertial frame of reference and $\nu$ the kinematic fluid viscosity ( $E^{-1}$ is the reciprocal microscale Ekman number). This is plainly independent of the requirement $d / L \ll 1$. We note in passing that, in the phenomenological analysis of the behaviour of local materials, $d$ does not appear as such and, since the concept of an idealized 'contact force' for the stress in a local material implies $d \rightarrow 0$, both requirements are satisfied simultaneously and the AMFI holds (though the double nature of this assumption is not generally made explicit).

For the kinetic theory of a hard-sphere gas, $d$ may be taken as the mean free path and the analogous group is that obtained by Lumley (1970) by considering the motion of the molecules. He shows that it is negligibly small under ordinary conditions, thus justifying the AMFI for a hard-sphere gas. The same conclusion is not valid for a 
suspension, however. In particular, the length of the Taylor column in the foregoing example is proportional to $E^{-1}$ (Greenspan 1968), and the quantity $\left(\mu^{*}-\mu_{2}\right) / \mu_{2} \phi$ is thus a function of $E^{-1}$ (presumably increasing).

Hence, the AMFI is valid for a suspension-like material only if the reciprocal Ekman number of the microscale motion $E^{-1}=\Omega d^{2} / \nu$ is small enough, where $\Omega$ characterizes the angular velocity of the rigid-body rotation of a macro-element relative to an inertial frame of reference. This rotation may exist due to vorticity of the macro-element or rotation of the material as a whole, or both.

Consider now the second form of the AMFI, the invariance of the constitutive equation to motion of observer. An important distinction must be drawn at this point: plainly, material behaviour and properties cannot depend upon the motion of an observer, but this affords no a priori ground to suppose that the description of such propertias is similarly invariant. In particular, the functional form of the constitutive equation appropriate for a given observer might be expected to depend upon his motion relative to an inertial frame (in an analogous fashion to the modification of the equation of motion for such an observer).

For a suspension with a non-zero microscale, $d$, such is indeed the case. In analysing the microdynamics which gives rise to the constitutive behaviour, a non-inertial observer must, of course, include 'fictitious' body forces by virtue of his own motion, and these are not frame-indifferent. In consequence, if $\bar{\Omega} d^{2} / \nu$ is significantly different from zero (where $\bar{\Omega}$ is now the observer's rotation relative to an inertial frame) the form of the constitutive equation will be significantly affected. Thus, if $d \neq 0$, it is clear that for a sufficiently non-inertial observer the AMFI is always invalid.

This conclusion is at first sight worrying since we may always postulate an observer who accelerates arbitrarily rapidly, and this suggests that the AMFI is of no practical usefulness. In fact, however, to choose such an observer is merely perverse: the inclusion of fictitious body forces in the macroscopic equation of motion, and the modification of the constitutive equation are precisely what are needed to nullify the effect of his acceleration and so have no effect on the material behaviour. In other words, provided that the first form of the AMFI holds, so that micro-inertia is negligible for accelerations of the suspension relative to an inertial frame $\left(E^{-1}=\Omega d^{2} / \nu \ll 1\right)$, consideration of the effect on the constitutive equation of fictitious forces which would be included by a non-inertial observer is unnecessary, and we may put $d=0$ for convenience. Only then does the AMFI become valid in its second form. In this sense, the 'invariance to observer' is clearly less fundamental than the first, 'invariance to rigid motion' form of the AMFI.

Now, considering flow of a suspension, we note that in most practical cases the magnitude of the vorticity is of the same order as the magnitude of the strain rate, and therefore $E^{-1}$ is comparable to the Reynolds number for the microscale flow. Thus the AMFI is not applicable in the situation considered in the main body of the present paper, nor in the 'small inertia' analysis of Lin et al. (1970).

Barthés-Biesel \& Acrivos (1973) have attempted to generalize the latter results to other linear flows. Their assumption that the Jaumann derivative is appropriate to describe the time evolution of the structure must be called into question, however. Doubtless, their results provide a useful approximation, but the invalidity of the AMFI for systems with micro-inertia indicates that use of the Jaumann derivative may not be adequate. 
In the most common case of a suspension with negligible micro-inertia $(R \ll 1)$ the AMFI is valid except perhaps if the suspension is in rapid rotation as a whole. We can estimate the order of the angular velocity at which the effect of rotation may become noticeable. For particles with $d \approx 10^{-1} \mathrm{~cm}$ in water $\left(\nu \approx 10^{-2} \mathrm{~cm}^{2} \mathrm{~s}^{-1}\right)$ we get $E^{-1}=1 \mathrm{~s} \times \Omega$, which means that very moderate rotation of $1 \mathrm{~s}^{-1}$ is enough for an appreciable effect while a rotation of $100 \mathrm{~s}^{-1}$ may cause drastic changes in the quantity $\left(\mu^{*}-\mu_{2}\right) / \mu_{2} \phi$.

The authors wish to thank Professor L. G. Leal, Professor W. R. Schowalter, Professor G. Astarita, Dr E. J. Hinch and Professor G. K. Batchelor for their helpful remarks concerning the applicability of the AMFI.

\section{REFERENCES}

Ames, W. F. 1969 Numerical Methods for Partial Differential Equations. New York: Barnes and Noble.

Astarita, G. \& Marrucci, G. 1974 Principles of Non-Newtonian Fluid Mechanics. MeGrawHill.

Barthés-Biesel, D. \& Acrivos, A. 1973 The rheology of suspensions and its relation to phenomenological theories for non-Newtonian fluids. Int. J. Multiphase Flow 1, 124.

Batchelor, G. K. 1967 An Introduction to Fluid Dynamics. Cambridge University Press.

BATChELOR, G. K. 1970 The stress system in a suspension of force-free particles. J. Fluid Mech. 41, 545-570.

BATCHELOR, G. K. 1971 The stress generated in a non-dilute suspension of elongated particles by pure straining motion. J. Fluid Mech. 46, 813-829.

Batchelor, G. K. 1974 Transport properties of two-phase materials with random structure. Ann. Rev. Fluid Mech. 6, 227-255.

Bird, R. B., Armstrong, R. C. \& Hassager, O. 1977 Dynamics of Polymeric Liquids. Vol. 1. Fluid Mechanics. Wiley.

Brenner, H. \& CondifF, D. W. 1974 Transport mechanics in systems of orientable particles. IV. Convective Transport. J. Colloid Interface Sci. 47, 199-264.

Dorodntsyy, A. A. \& Meliek, N. A. 1968 Approaches to the solution of stationary NavierStokes equations. U.S.S.R. Comput. Math. \& Math. Phys. 8 (2), 205-217.

Greenspan, H. P. 1968 The Theory of Rotating Fluids. Cambridge University Press.

Grosch, C. E. \& OrszaG, S. A. 1977 Numerical solution of problems in unbounded regions: coordinate transforms. J. Comp. Phys. 25, 273-296.

Hinch, E. J. \& Leal, L. G. 1975 Constitutive equations in suspension mechanies. Part 1. General formulation. J. Fluid Mech. 71, 481-495.

Hinch, E. J. \& Leal, L. G. 1976 Constitutive equations in suspension mechanics. Part 2. Approximate forms for a suspension of rigid particles affected by Brownian rotations. $J$. Fluid Mech. 76, 187-208.

Israfir, M. 1970 A fast implicit numerical method for time dependent viscous flows. Stud. Appl. Math. 49, 327-349.

JEFFREY, D. J. 1974 Group expansions for the bulk properties of a statistically homogeneous random suspension. Proc. Roy. Soc. A 338, 503-516.

JEFFREY, D. J. 1977 The physical significance of non-convergent integrals in expressions for effective transport properties. Proc. 2nd Int. Symp. on Continuum Models of Discrete Systems, pp. 653-674. University of Waterloo Press.

JEFFREY, D. J. \& ACRIvos, A, 1976 The rheological properties of suspensions of rigid particles. A.I.Ch.E. J. 22, 417-432.

Lin, C. J., Peery, J. H. \& Schowalter, W. R. 1970 Simple shear flow round a rigid sphere: inertial effects and suspension rheology. J. Fluid Mech. 44, 1-17. 
Lumley, J. L. 1970 Toward a turbulent constitutive relation. J. Fluid Mech. 41, 413-434.

MCConnelu, A. J. 1957 Applications of Tensor Analysis. Dover.

O'Brien, R. W. 1979 A method for the calculation of the effective transport properties of suspensions of interacting particles. J. Fluid Mech. 91, 17-39.

Pearson, J. R. A. 1959 The effect of uniform distortion on weak homogeneous turbulence. J. Fluid Mech. 5, 274-288.

Rivkind, V. Ya. \& RYskin, G. 1976 Flow strudture in motion of a spherical drop in a fluid medium at intermediate Reynolds numbers. Fluid Dynamics 11, 5-12.

Rivkind, V. Ya., Ryskin, G. \& Fishbein, G. A. 1976 Flow around a spherical drop at intermediate Reynolds numbers. Appl. Math. \& Mech. (PMM) 40, 687-691.

RoAche, P.J. 1972 Computational Fluid Dynamics. Albuquerque: Hermosa.

Schowalter, W. R. 1978 Mechanics of Non-Newtonian Fluids. Pergamon.

Serrin, J. 1959 Mathematical principles of classical fluid mechanics, Handbuch der Physik, vol. 8/1, pp. 125-263. Springer.

TAylor, G. I. 1934 The formation of emulsions in definable fields of flow. Proc. Roy. Soc. A 146, 501-523.

Truesderl, C. 1977 A First Course in Rational Continuum Mechanics, Vol. 1. General Concepts. Academic Press.

Truesdel., C. \& Nolt, W. 1965 The Non-Linear Field Theories of Mechanics, Handbuch der Physik, vol. 3/3. Springer. 